\title{
Nature Medicine, reloaded
}

\begin{abstract}
Molecular medicine has undergone profound changes since the publication of the first issue of Nature Medicine 15 years ago this month. To keep up with these changes, we strengthen our commitment to publishing the best research and the most topical newsand commentary on translational medicine by adding more pages to the journal.
\end{abstract}

$N$ ature Medicine's inaugural editorial, published in January 1995, stated that the journal would be "home to papers that bridge the gap between cutting edge biological research and more clinically oriented human investigation." In current parlance, Nature Medicine was to be the home of translational research.

Fifteen years later, our mission remains the same. What has changed is the landscape in which biomedical research is conducted. For starters, funding organizations worldwide now pay a premium for 'translational projects' - research that, broadly speaking, aims to satisfy an unmet medical need. As a result, our niche has expanded, we serve a bigger community and translational research is more visible than ever.

The drug discovery model has also changed profoundly over the past 15 years. Although there have been some stunning success stories, most of the discoveries made at the lab bench continue to fall short on their way to the bedside. Some pharmaceutical companies have reacted to this trend by becoming more averse to risk, whereas others have actually increased their wagers on early-stage academic research. What most firms seem to agree about is the need to diversify their pipeline, moving away from the entrenched, small-molecule-based model of drug discovery to embrace ideas such as personalized medicine and the potential use of stem cells, gene therapy and RNA interference to treat disease.

Many other important changes over the past two decades have affected the way translational research is conducted. Projects are increasingly carried out by large consortia, forcing us to think about new ways of giving due credit to authors. Systems biology approaches have made it commonplace to generate large amounts of data, which are harder and harder to make sense of. We are much more sensitive to conflicts of interest and their potentially nefarious influence on research.

The advent of the Internet has also transformed the way in which scientists communicate their research. Perhaps as importantly, the Internet has given the general public unprecedented access to scientific and medical information. Reliable websites with up-to-date and accurate scientific information and analysis have therefore become more necessary than ever.
These and other changes in the translational research landscape have directly affected our views on what we consider for publication in Nature Medicine. The larger size of the translational community means that we receive increasingly more submissions. The introduction of systems biology approaches to biomedicine has resulted in more and more studies on disease biomarkers, forcing us to think more deeply about how to make sense of these contributions. And with the plethora of submitted studies that claim to have identified a molecule that is "critically important" for essentially any disease you can think of, it is harder than ever to identify those that report a true disease target that will eventually result in the discovery of a new therapy.

We are aware of our visibility in the translational community and wish to strengthen our position as a leading source of news, commentary and research in biomedicine. After 15 years of serving our readers, we wish to renew our commitment to offering you the best studies that "bridge the gap between cutting edge biological research and more clinically oriented human investigation" and to being a trusted source of information on every facet of the biomedical world.

To that end, we are pleased to announce that, starting with this issue, Nature Medicine is growing. We have recruited additional news staff to increase our news coverage in print and online, building on our existing podcast, Twitter (http://twitter.com/ NatureMedicine) and Facebook (http://go.nature.com/mYn63n) presence. We will also increase the number and scope of our News and Views, Commentaries and Reviews by appointing an extra editor dedicated full time to this section. Having this extra member on the team will enable us to introduce new sections that, we hope, will be of interest to the translational research community and will reflect the changing nature of our discipline. Last, an additional editor for research manuscripts will help us cope with our ever-growing submissions load, enabling us to provide you, our authors, with decisions as quickly and as fairly as possible.

In short, we want to renew our commitment to being the home of translational research. As always, we welcome your input on the direction the journal is taking and thank those of you who have taken the time to share your thoughts with us. 